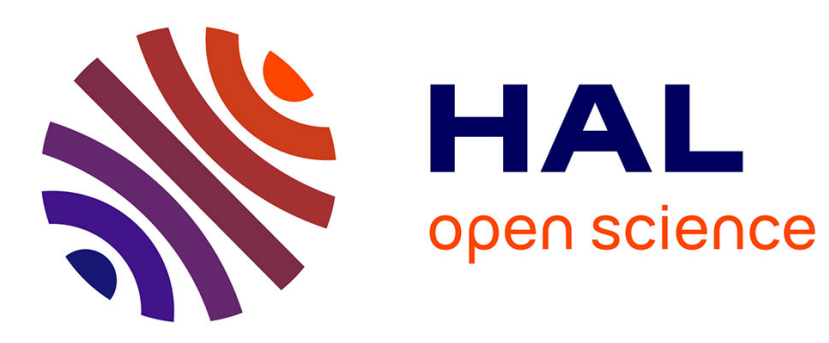

\title{
Acoustic noise of electromagnetic origin in a fractional-slot induction machine
}

Jean Le Besnerais, Vincent Lanfranchi, Michel Hecquet, Pascal Brochet, Guy Friedrich

\section{- To cite this version:}

Jean Le Besnerais, Vincent Lanfranchi, Michel Hecquet, Pascal Brochet, Guy Friedrich. Acoustic noise of electromagnetic origin in a fractional-slot induction machine. COMPEL: The International Journal for Computation and Mathematics in Electrical and Electronic Engineering, 2008. hal-01730150

\author{
HAL Id: hal-01730150 \\ https://hal.science/hal-01730150
}

Submitted on 13 Mar 2018

HAL is a multi-disciplinary open access archive for the deposit and dissemination of scientific research documents, whether they are published or not. The documents may come from teaching and research institutions in France or abroad, or from public or private research centers.
L'archive ouverte pluridisciplinaire HAL, est destinée au dépôt et à la diffusion de documents scientifiques de niveau recherche, publiés ou non, émanant des établissements d'enseignement et de recherche français ou étrangers, des laboratoires publics ou privés. 


\title{
Acoustic noise of electromagnetic origin in a fractional-slot induction machine
}

\author{
Jean Le Besnerais*, Vincent Lanfranchi ${ }^{\dagger}$, Michel Hecquet*, Pascal Brochet* and Guy Friedrich ${ }^{\dagger}$ \\ * L2EP - Ecole Centrale de Lille, Villeneuve d'Ascq, France (jean.le_besnerais@centraliens.net) \\ $\dagger$ LEC - UTC, Compiègne, France (vincent.lanfranchi@utc.fr)
}

\begin{abstract}
Purpose - The main purpose of this paper is to apply a fast analytical model of the acoustic behaviour of pulse-width modulation (PWM) controlled induction machines (Besnerais, Fasquelle, Hecquet, Lanfranchi \& Brochet 2006) to a fractionalslot winding machine. A second goal is to analytically clarify the interaction between space harmonics and time harmonics in audible electromagnetic noise spectrum.

Methodology/approach - A multilayer single-phase equivalent circuit calculates the stator and rotor currents. Air-gap radial flux density, which is supposed to be the only source of acoustic noise, is then computed with winding functions formalism. Mechanical and acoustic models are based on a 2D ring stator model. A method to analytically derive the orders and frequencies of most important vibration lines is detailed. The results are totally independent of the supply strategy and winding type of the machine. Some variable-speed simulations are run on a $700 \mathrm{~W}$ fractional-slot induction machine in sinusoidal case as a first validation of theoretical results.

Findings - The influence of both winding space harmonics and PWM time harmonics on noise spectrum is exposed. Most dangerous orders and frequencies expressions are demonstrated in sinusoidal and PWM cases. For traditional integral windings, it is shown that vibration orders are necessarily even. When stator slot number is not even, which is the case for fractional windings, some odd order deflection appear: the radial electromagnetic power can therefore dissipate as vibrations through all stator deformation orders, leading to a potentially lower noise level at resonance.

Research limitations - The analytical work of this paper does not consider saturation and eccentricity harmonics which can play a significant role in noise radiation.

Practical implications - The analytical model and theoretical results presented in this paper help designing low-noise induction machines, and diagnosing noise or vibration problems.

Originality/value - This paper details a fully analytical acoustic and electromagnetic model of a PWM fed induction machine, and demonstrate the theoretical expression of main noise spectrum lines combining both time and space harmonics.

Keywords - Induction machine, Magnetic noise, Vibrations, Fractional-slot winding.

Paper type - Research paper.
\end{abstract}




\section{NOMENCLATURE}

\section{Electrical notations}

$f_{1} \quad$ Fundamental stator supply frequency

$f_{m n}^{r} \quad$ Rotor current $n$-th time and $m$-th space harmonic

$f_{n}^{s} \quad$ Stator current $n$-th time harmonic

$f_{m m} \quad$ Magnetomotive force

$f_{R} \quad$ Rotor shaft rotation frequency $\left(f_{R}=(1-s) f_{1} / p\right)$

$F_{r}, F_{s} \quad$ Rotor and stator mmf waves

$g \quad$ Air-gap width

$h_{r}, h_{s} \quad$ Integers involved in rotor and stator $\mathrm{mmf}$

space harmonics expression

$i_{b}^{r} \quad b$-th rotor bar current

$i_{q}^{s} \quad q$-th stator phase current

$k_{r}, k_{s} \quad$ Integers representing the rotor and stator slotting terms in the permeance Fourier series expansion

$l_{s d} \quad$ Stator tooth width

$l_{\text {se }} \quad$ Stator slot opening

$l_{r d} \quad$ Rotor tooth width

$l_{\text {re }} \quad$ Rotor slot opening

$L_{n} \quad$ Force line number $n$

$m \quad m$-th space harmonic induced by stator winding in rotor bars currents (fundamental $m=p$ )

$n \quad n$-th time harmonic generated by PWM supply

(fundamental $n=1$ )

$p \quad$ Number of pole pairs

$P_{r}, P_{s} \quad$ Rotor and stator slotting permeance waves

$q_{s} \quad$ Number of stator phases

$s_{m n} \quad$ Harmonic slip (fundamental $s_{p 1}=s$ )

$U_{n} \quad n$-th time harmonic of PWM phase voltage

$Z_{r} \quad$ Number of rotor slots

$Z_{s} \quad$ Number of stator slots

$\alpha_{r} \quad$ Rotor angular position in stator steady frame

$\alpha_{r}^{k} \quad k$-th rotor slot angular position

$\alpha_{s} \quad$ Angular position in stator steady frame

$\alpha_{s}^{k} \quad k$-th stator slot angular position

$\beta_{r} \quad$ Rotor slot half angular width

$\epsilon_{r}, \epsilon_{s} \quad \pm 1$ factor representing rotor or stator mmf wave direction

$\eta_{i j} \quad \pm 1$ factor resulting from the interaction of fields $i$ and $j$

$\Lambda \quad$ Permeance per unit area

$\nu_{s}, \nu_{r} \quad$ Stator and rotor mmf space harmonics

$\omega_{n} \quad n$-th time harmonic pulsation coming from PWM

$\theta_{r}^{k} \quad$ Angular position of $k$-th rotor tooth

$\Xi_{m} \quad$ Winding factor of $m$-th space harmonic 
Mechanical and acoustic notations

$D_{c} \quad$ Stator outer radius (without frame)

$f \quad$ Exciting force frequency

$f_{m} \quad m$-th mode natural frequency

$h_{c} \quad$ Height of stator yoke

$L_{r} \quad$ Rotor stack length

$L_{s} \quad$ Stator stack length

$m \quad m$-th circumferential spatial mode

$R_{a} \quad$ Stator bore radius

$R_{c} \quad$ Stator mean radius (computed without the teeth)

$R_{e} \quad$ Stator core outer radius

$R_{i} \quad$ Stator slots bottom radius

$Y_{m \omega}^{d} \quad$ Dynamic deflection of order $m$ at pulsation $\omega$

$Y_{m \omega}^{s} \quad$ Static deflection of order $m$ at pulsation $\omega$

$\xi_{m} \quad m$-th mode damping coefficient

\section{INTRODUCTION}

As acoustic norms become stricter in power electrical transport systems, the understanding and prediction of the noise of electromagnetic origin at variable speed is crucial. In fact, the Pulse-Width Modulation (PWM) strategies add many harmonics to the air-gap Maxwell forces spectrum, leading to possibly harmful noise and vibrations. Stator windings induce in rotor currents additional time harmonics which can also significantly enrich the electromagnetic forces spectrum, especially when running at high slip. Audible electromagnetic noise spectrum therefore results from a complex combination of both PWM time harmonics and winding space harmonics. Predicting this so-called "magnetic noise" level requires to precisely model both the mechanical structure of the machine and its electromagnetic excitation.

This paper presents a simulation tool of the PWM-fed induction machine, DIVA (Ait-Hammouda 2005, Besnerais et al. 2006), which is able to consider the whole space and time harmonics involved in magnetic noise generation without a prohibitive computation time. The analytical derivation of main radial force lines spatial modes and frequencies is exposed, including the influence of stator winding and rotor bars space harmonics. Finally, some variable-speed simulations are presented and analysed on the ground of these theoretical results. The acoustic role of odd spatial modes will be also discussed.

The motor studied in this paper is a $700 \mathrm{~W}$ three-phase squirrel-cage induction machine with $p=2$ pole pairs, $Z_{r}=21$ rotor bars and $Z_{s}=27$ stator slots. Its double-layer winding is a fractional-slot winding as its number of slot per pole and per phase is not an integer.

\section{Electromagnetic MOdel}

\section{A. Currents computation}

Supply phase voltage can be either given by experimental data or computed analytically. Then, stator and rotor phase currents are computed using an extension of the fundamental single-phase equivalent circuit, including all space and time harmonics (Hubert 2000). As illustrated in Fig. 1, at each time harmonic $U^{n}$ of frequency $f_{n}^{s}$ coming from the PWM supply phase voltage corresponds an equivalent circuit including the influence of $m^{1}$ stator winding space harmonics.

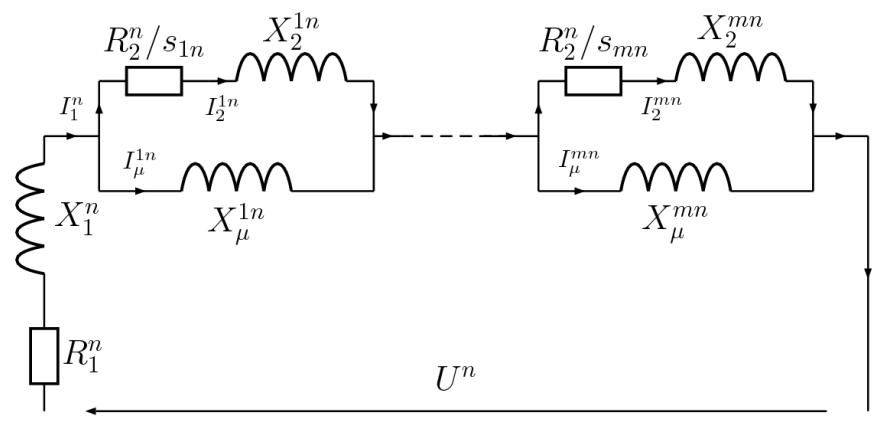

Fig. 1. Multi-layer single phase equivalent circuit

The equations of this equivalent circuit consist in $n$ global mesh equations

\footnotetext{
${ }^{1}$ We define the space harmonics as the "spatial frequencies" coming from the Fourier Transform of a function of the mechanical angle, not of the electrical angle. With such a definition, the space fundamental is the number of pole pairs $p$ and not 1 .
} 


$$
\begin{gathered}
U^{n}=\left(R_{1}^{n}+j X_{1}^{n}\right) I_{1}^{n}+\sum_{m} j X_{\mu}^{m n} I_{\mu}^{m n} \\
\doteq Z_{1}^{n} I_{1}^{n}+\sum_{m} Z_{\mu}^{m n} I_{\mu}^{m n}
\end{gathered}
$$

$m \times n$ node equations

$$
I_{1}^{n}=I_{\mu}^{m n}+I_{2}^{m n}
$$

and $m \times n$ local mesh equations

$$
\begin{gathered}
0=j X_{\mu}^{m n} I_{\mu}^{m n}-\left(j X_{2}^{m n}+R_{2}^{n} / s_{m n}\right) I_{2}^{m n} \\
\doteq Z_{\mu}^{m n} I_{\mu}^{m n}-Z_{2}^{m n} I_{2}^{m n}
\end{gathered}
$$

$R_{1}^{n}$ and $R_{2}^{n}$ respectively stand for the stator and rotor equivalent resistances which depend on time harmonics because of skin effect. The detailed expression of this effect can be found for instance in (Matsuse, Hayashida, Kubota \& Yoshida 1994).

$X_{1}^{n}=\omega_{n} L_{1}=2 \pi f_{s}^{n} L_{1}$ is the stator total phase reactance, $L_{1}$ being the corresponding inductance. $X_{2}^{m n}=\omega_{n} L_{2}^{m}$ is the rotor total reactance, $L_{2}^{m}$ being the rotor inductance refered to the primary. It depends on space harmonics $m$ because it is proportional to the transformation factor which is a function of the winding distribution factors $\Xi_{m}$. The expression of these distribution factors can be found for instance in (Salminen 2004) for both integral and fractional-slot windings.

$X_{\mu}^{m n}=\omega_{n} L_{\mu}^{m}$ is the magnetizing reactance in which we can eventually include a parallel iron losses equivalent resistance. $L_{\mu}^{m}$ is a generalization of the fundamental magnetizing inductance $L_{\mu}^{p}$ (Hubert 2000)

$$
L_{\mu}^{m}=L_{\mu}^{p}\left(\frac{p}{m} \frac{\Xi_{m}}{\Xi_{p}}\right)^{2}
$$

where $\Xi_{m}$ is the winding factor of the $m$-th space harmonic generated by the stator winding.

$I_{2}^{m n}$ and $I_{1}^{n}$ are the harmonic rotor and stator currents, $I_{\mu}^{m n}$ is the harmonic magnetizing current. $s_{m n}$ is the harmonic slip

$$
s_{m n}=1 \pm \frac{m \omega_{1}}{p \omega_{n}}(1-s)
$$

where $s$ is the fundamental slip and the \pm 1 factor takes into account the propagation direction of harmonic fields induced by stator currents.

For a given time harmonic $n, 2 m+1$ equations can be grouped in the following matrix form

$$
\mathbf{Z}^{\mathbf{n}} \cdot \mathbf{I}^{\mathbf{n}}=\mathbf{U}^{\mathbf{n}}
$$

with

$$
\mathbf{Z}^{\mathbf{n}}=\left(\begin{array}{ccc}
Z_{1}^{n} & \mathbf{Z}_{\mu}^{n} & \mathbf{0} \\
\mathbf{1} & -\mathbb{I} & -\mathbb{I} \\
\mathbf{0} & \mathbf{D}_{\mu}^{n} & -\mathbf{D}_{2}^{n}
\end{array}\right) \quad \mathbf{I}^{\mathbf{n}}=\left(\begin{array}{c}
I_{1}^{n} \\
\mathbf{I}_{\mu}^{n} \\
\mathbf{I}_{2}^{n}
\end{array}\right) \quad \mathbf{U}^{\mathbf{n}}=\left(\begin{array}{c}
U^{n} \\
\mathbf{0} \\
\mathbf{0}
\end{array}\right)
$$

where $\mathbf{1}$ is the unitary vector, $\mathbb{I}$ the identity matrix, $\mathbf{0}$ the null matrix, $\mathbf{Z}_{\mu}^{n}$ the $Z_{\mu}^{m n}$ line vector, $\mathbf{D}_{\mu}^{n}$ the $Z_{\mu}^{m n}$ diagonal matrix and $\mathbf{D}_{2}^{n}$ the $Z_{2}^{m n}$ diagonal matrix, $\mathbf{I}_{2}^{n}$ the $I_{2}^{m n}$ column vector and $\mathbf{I}_{\mu}^{n}$ the $I_{\mu}^{m n}$ column vector. System (6) is solved for each non-zero time harmonic $U^{n}$.

\section{B. Air-gap radial flux density computation}

Radial air-gap flux density $B_{g}$ is expressed as

$$
B_{g}\left(t, \alpha_{s}\right)=\Lambda\left(t, \alpha_{s}\right) f_{m m}\left(t, \alpha_{s}\right)
$$

where $\alpha_{s}$ is the angular position in the stator steady frame, $\Lambda=\mu_{0} / g_{e}$ is the air-gap permeance per unit area, $g_{e}$ being the effective air-gap width (detailed in section V-A) and $\mu_{0}$ the air-gap magnetic permeability, and $f_{m m}$ is the total magnetomotive force (mmf). Applying the Ampere's law to an appropriate path, one can show (Bossio, Angelo, Solsona, Garcia \& Valla 2004) that

$$
f_{m m}\left(t, \alpha_{s}\right)=\underbrace{\sum_{q=1}^{q_{s}} i_{q}^{s}(t) N_{q}^{s}\left(\alpha_{s}\right)}_{f_{m m}^{s}}+\underbrace{\sum_{b=1}^{Z_{r}} i_{b}^{r}(t) N_{b}^{r}\left(t, \alpha_{s}\right)}_{f_{m m}^{r}}
$$



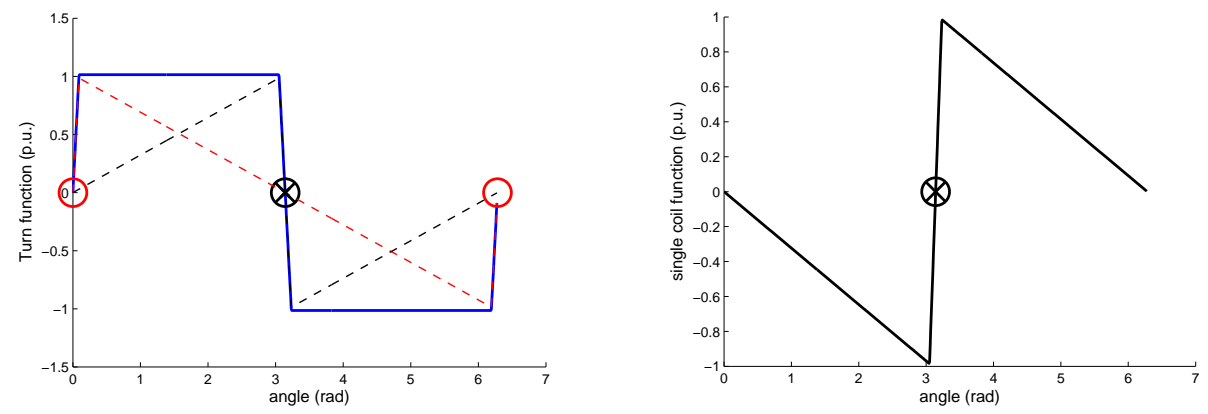

Fig. 2. Normalized full-pitch winding distribution function $N_{q}^{s}$ (left) and the equivalent normalized coil distribution function (right).

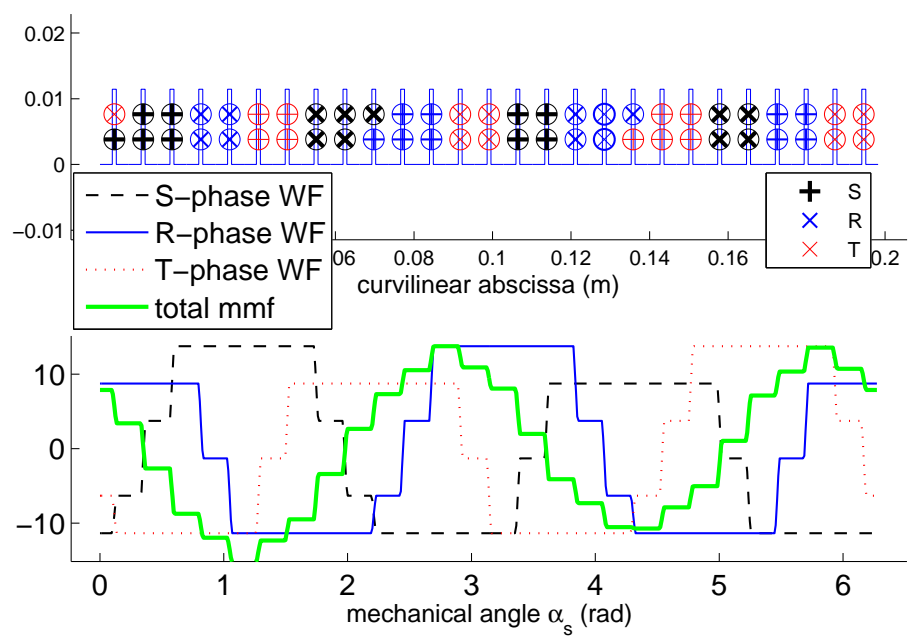

Fig. 3. Winding functions and total stator magnetomotive force at a given time

where $N_{q}^{s}$ is the 2-D turns function (TF) or winding distribution function associated to the stator $q$-th phase with current $i_{q}^{s}$, and $N_{b}^{r}$ is the turns function associated to the rotor $b$-th bar with current $i_{b}^{r}$. Note that contrary to stator TFs, rotor TFs are time-dependent.

Normalized phase TF of a full-pitch winding is represented in FIG. 2 including a linear rise in slots. We can see that it can be computed summing some equivalent coil turns function weighted with current sign. That decomposition and the winding function formalism have been chosen to be implemented in DIVA because they allow modelling any type of winding, especially fractional-slot ones. Phase TFs and the resulting stator mmf of the double-layer fractional-slot test motor are displayed in FIG. 3 .

In order to compute the squirrel-cage rotor mmf, one has to consider the rotor cage as an equivalent $Z_{r}$-phase circuit with $Z_{r}$ loop currents (Henao, Razik \& Capolino 2005, Joksimovic, Djurovic \& Penman 2001). In that case, the turns functions can still be used but with an equivalent number of turns of 1.

Stator and rotor mmf of our test motor as well as their space harmonic content are shown in FIG. 4 in sinusoidal on-load case, with a $3.05 \%$ slip. We can see that the rotor mmf wave tends to counterbalance the stator mmf wave as predicted by the Lenz law. Besides fundamental at $\nu_{r}=p$, rotor mmf contains main space harmonics $\nu_{r}=19$ and 23 corresponding to $\nu_{r}=Z_{r} \pm p$. The same analysis can be carried with stator mmf which containts the main space harmonics $\nu_{s}=Z_{s} \pm p$. Spectral content of stator and rotor mmf is detailed later in section V-B.

\section{Validation}

Radial air-gap flux density has been compared to finite element method (FEM) simulations for different shorted-pitch machines, supply frequencies and voltages. Motor torque and phase current were also validated with FEM and experiments in on-load case (Besnerais et al. 2006). 

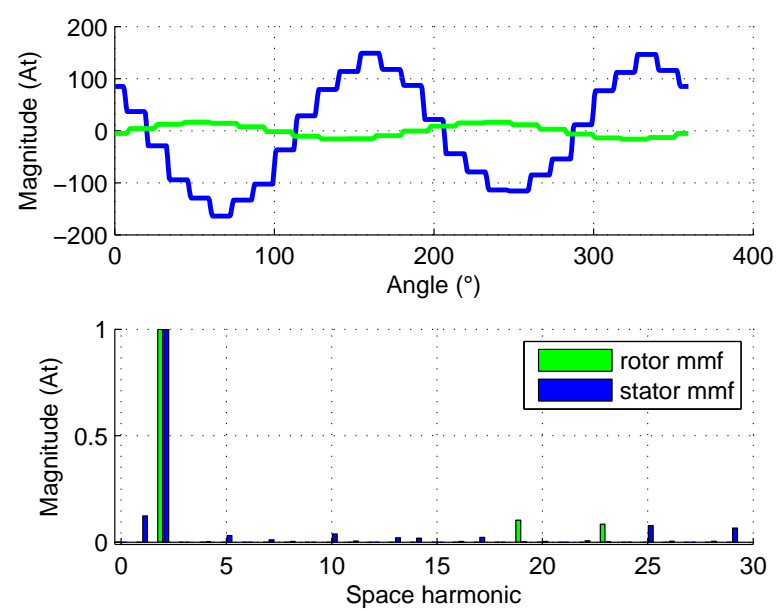

Fig. 4. Rotor and stator mmf as a function of mechanical angle and their normalized space harmonic content at a given time.

\section{MECHANICAL MODEL}

\section{A. Exciting force computation}

Neglecting the tangential component of the Maxwell tensor and the magnetostrictive effect, the exciting pressure $P_{M}$ responsible for magnetic noise can be approximated by

$$
P_{M}=\frac{B_{g}^{2}}{2 \mu_{0}}
$$

\section{B. Stator deflections computation}
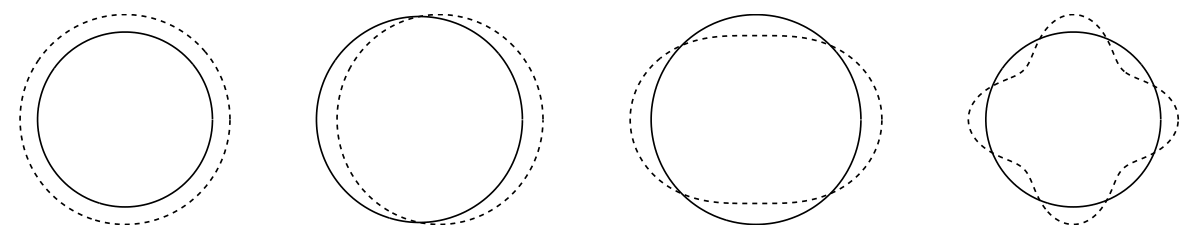

Fig. 5. Shape of circumferential spatial modes $m=0, m=1, m=2$ and $m=4$.

The mechanical model assumes that the stator is 2D ring with free-free boundary conditions. Therefore, only the in-plane circumferential spatial modes $m$ are considered (see FIG. 5). However, we will see that the third dimension is taken into account through the rotor bending motion. Static deflections $Y_{m \omega}^{s}$ are first computed in function of the complex amplitudes $P_{m \omega}$ of the 2-D discrete Fourier transform of radial pressure $P_{M}$. Their amplitudes are found using the theory for a simply supported beam carrying a sinusoidally ditributed load (Jordan 1950). For $m=0$,

$$
Y_{0 \omega}^{s}=P_{0 w} \frac{R_{c} R_{a}}{E_{c} h_{c}}
$$

where $h_{c}$ is the thickness of the stator back, $R_{c}$ is the mean stator radius (computed without considering the teeth), $R_{a}$ is the stator bore radius and $E_{c}$ is the stator Young modulus. $Y_{1 \omega}^{s}$ is generated by the force per unit area $P_{1 \omega}$ which excites the rotor (cf. FIG. 5), it corresponds to a bending motion in longitudinal direction. Such a deflection can be approximated considering the rotor as a simply supported beam loaded with pressure $P_{1 \omega}$ :

$$
Y_{1 \omega}^{s}=P_{1 \omega} \frac{4 R_{a} l_{s h}^{3} L_{r}}{3 E_{s h} D_{s h}^{4}}
$$

where $L_{r}$ stands for rotor length, $E_{s h}$ for rotor shaft Young modulus, $D_{s h}$ for its diameter and $l_{s h}$ for the distance between bearings. Finally, for orders $m \geq 2$,

$$
Y_{m \omega}^{s}=P_{m \omega} \frac{12 R_{a} R_{c}^{3}}{E_{c} h_{c}^{3}\left(m^{2}-1\right)^{2}}
$$


Then, dynamic deflections $Y_{m \omega}^{d}$ are computed as

$$
Y_{m \omega}^{d}=Y_{m \omega}^{s}\left[\left(1-f^{2} / f_{m}^{2}\right)^{2}+4 \xi_{m}^{2} f^{2} / f_{m}^{2}\right]^{-1 / 2}
$$

where $\xi_{m}$ is the modal damping coefficient, and $f_{m}$ is the $m$-th mode natural frequency. $\xi_{m}$ can be computed using the experimental law established by (Yang 1981)

$$
2 \pi \xi_{m}=2.76 \times 10^{-5} f_{m}+0.062
$$

In (13) the second order magnification factor comes from the fact that any excited system motion is ruled by a second order differential equation. The damping coefficient quantifies how much kinetic energy decays through Coulomb and viscous frictions, which mainly occur in lamination, windings and insulation (Verma \& Balan 1998). This filter also models the resonance phenomenon: a dynamic deflection of mode $m$ is the highest when the exciting frequency $f$ is the closest from the natural frequency $f_{m}$. At the resonance, the dynamic deflection is given by

$$
Y_{m \omega}^{d}=\frac{Y_{m \omega}^{s}}{2 \xi_{m}}
$$

Thus, the more there are damping materials, the lower vibrations occur. From (10) and (12) it can be also deduced that

$$
\frac{Y_{m \omega}^{d}}{Y_{0 \omega}^{d}} \propto \frac{P_{m \omega}}{P_{0 \omega}} \frac{R_{c}^{2}}{h_{c}^{2}}
$$

Therefore, $R_{c} / h_{c}$ ratio sizes the ability of the stator to radiate vibrations : the higher $R_{c}$ is and the lower $h_{c}$ is, the larger and the thinner the stator is, and the more it plays the role of sound box. Nevertheless, decreasing $R_{c}$ at constant $h_{c}$ does not necessarily decrease noise because stator radiation efficiency increases with $L_{f} / R_{f} \approx L_{s} / R_{c}$ ratio.

Analytical prediction of $f_{m}$ natural frequencies is a difficult task. In our case, the winding and teeth effects are taken into account by modifying $=$ stator mass density $\rho_{c}$, defining

$$
\rho_{c}^{\prime}=k_{s} \frac{M_{t}+M_{c}}{\pi L_{s}\left(R_{c}^{2}-R_{a}^{2}\right)}
$$

where $k_{s}$ is the stator stacking factor, $M_{t}$ the teeth mass, $M_{c}$ the stator yoke mass, $L_{s}$ the stator length, $R_{e}$ the stator core outer radius and $R_{i}$ the stator slots bottom radius. Windings and insulation mass was therefore neglected, which might be only applicable to small machines. Zero-th mode order natural frequency is

$$
f_{0}=\frac{1}{2 \pi R_{c}} \sqrt{\frac{E_{c}}{\rho_{c}^{\prime}}}
$$

Mode number 1 natural frequency can be approximated treating the rotor and its shaft as a simply supported beam with a ring (Maliti 2000). Shaft stiffness is

$$
K_{s h}=\frac{3 \pi E_{s h} D_{s h}}{4 l_{s h}^{3}}
$$

Modal mass $M_{r}$ equals rotor ring mass plus half shaft mass. We obtain:

$$
f_{1}=\frac{1}{2 \pi} \sqrt{\frac{K_{s h}}{M_{r}}}
$$

This is a very simple model as it does not consider rotor lamination stiffness and assumes that the bearings are rigid. $m$-th mode natural frequency $(m \geq 2)$ is

$$
f_{m}=f_{0} \Gamma \frac{m\left(m^{2}-1\right)}{\sqrt{m^{2}+1}} \quad \Gamma=\frac{h_{c}}{2 \sqrt{3} R_{c}}
$$

Finally, in order to take into account the three-dimensional effects, these frequencies $f_{m}$ are mutiplied by additional factors $K_{m}$ (Cremer, Heckl \& Ungar 1988): $K_{2}=1.14, K_{3}=1.04$ and $K_{4}=1.02$.

Notice that

$$
f_{m} \approx \frac{1}{R_{c}} \frac{h_{c}}{R_{c}}=\frac{h_{c}}{R_{c}^{2}}
$$

Thus, increasing $h_{c} / R_{c}$ ratio in order to limit vibrations will also increase stator natural frequencies. 
TABLE I

RESUlTS OF DIFFERENT METHODS FOR STATOR NATURAL FREQUENCIES COMPUTATION (Hz). OR: OUT OF RANGE, ND: NON DEFINITE.

\begin{tabular}{|c|c|c|c|c|}
\hline$m$ & Analytical & 2-D FEM & Shock Method & Sinus Method \\
\hline 0 & 14859 & 14656 & OR & OR \\
1 & 1100 & ND & 1200 & 1273 \\
2 & 2478 & 2364 & 2400 & 2423 \\
3 & 6396 & 6473 & 6100 & 6210 \\
4 & 12028 & 11898 & 11700 & OR \\
\hline
\end{tabular}

\section{Validation}

Induction machine natural frequencies have been calculated by 2-D FEM, and measured by shock and sinus methods (Hubert \& Friedrich 2002). Comparison with the analytical method are presented in Table I. There is a good agreement between analytical results and experiments, because test motor geometry is rather simple (thin circular frame). The analytical computation of mode 1 natural frequency is the most inaccurate, as bearings stiffness is not considered and the measurement of bearings distance was not very precise.

\section{ACOUSTIC MODEL}

\section{A. Sound power level computation}

Vibration velocity of order $m$ is then $v_{m \omega}=Y_{m \omega}^{d} 2 \pi f$. Sound power radiated by the vibrations of mode $m$ and frequency $f$ is

$$
W_{m}(f)=\frac{1}{2} \rho_{0} c_{0} S_{c} \sigma_{m}(f)<\overline{v_{m \omega}^{2}}>
$$

where $S_{c}$ is the stator outer surface, $\rho_{0}$ the air density, $c_{0}$ the speed of sound, and $\sigma_{m}$ the modal radiation efficiency. $\sigma_{m}$ is approximated using either its pulsating sphere expression or its infinite cylinder expression according to stator dimensions (Timar \& Lai 1994). 1/2 factor in (23) takes into account bakeward and forward-travelling vibration waves.

Sound power level at frequency $\mathrm{f}$ is

$$
L_{w}(f)=10 \log _{10}\left(\sum_{m} W_{m}(f) / W_{0}\right), \quad W_{0}=10^{-12} W
$$

A-weighted total sound power level is finally obtained as

$$
L_{w A}=10 \log _{10}\left(\sum_{f} 10^{0.1\left(L_{w}(f)+\Delta L_{A}(f)\right)}\right)
$$

where $\Delta L_{A}(f)$ shift is a function of human's ear sensitivity.

\section{B. Validation}

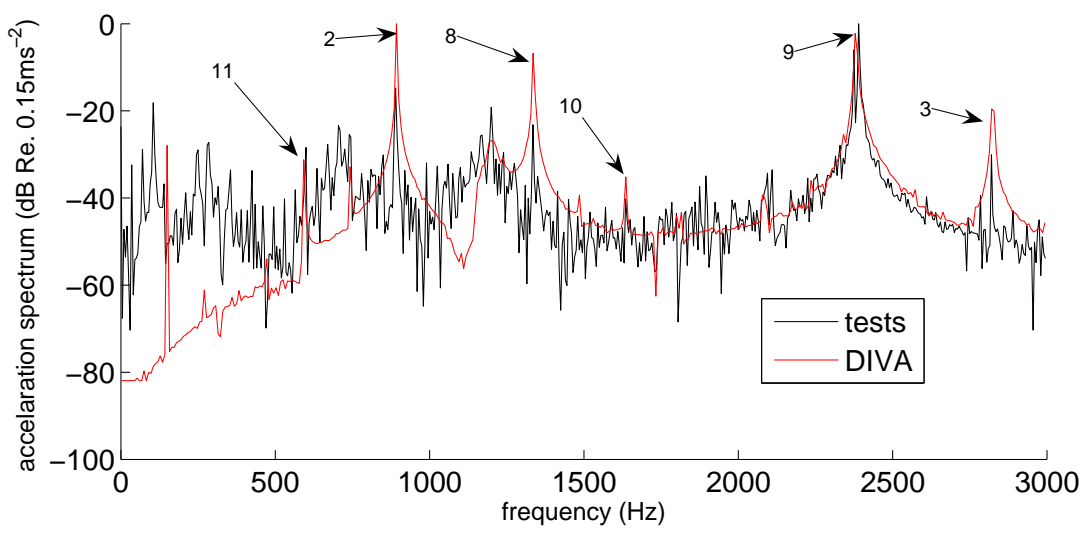

Fig. 6. Comparison between experimental and simulated acceleration spectra at $f_{s}=75 \mathrm{~Hz}$ and $s=5.6 \%$ in sinusoidal case.

FIG. 6 compares simulated and experimental stator frame acceleration spectra at supply frequency $f_{s}=75 \mathrm{~Hz}$, and slip $s=5.6 \%$, in sinusoidal case. High differences appear under $500 \mathrm{~Hz}$, which mainly come from mechanical vibrations that are 
not considerer in the model. We can see that the simulation tool correctly predicts main vibration lines. There expression can be found with analytical results of section $\mathrm{V}$ : for instance, the $2800 \mathrm{~Hz}$ line (number 3 ) is of order $1=3 Z_{s}-4 Z_{r}+2 p$ and frequency $f_{1}\left(4(1-s) \frac{Z_{r}}{p}-2\right)$, and the $900 \mathrm{~Hz}$ line (number 2) is of order $2=Z_{s}-Z_{r}-2 p$ and frequency $f_{1}\left((1-s) \frac{Z_{r}}{p}+2\right)$.

\section{ANALYTICAL EXPRESSION OF MAIN RADIAL FORCE SPECTRUM LINES}

This part aims at characterizing the electromagnetic noise spectral content which is the same for noise and vibration. The electromagnetic force spectrum results from the interaction of permeance and $\mathrm{mmf}$ spectra, which can be determined using Fourier series. However, airgap flux density is not computed in DIVA on the base of these Fourier series because they are computationally costly.

\section{A. Permeance orders and frequencies}

The Fourier series of permeance per unit area is (Brudny 1997, Hesse 1992)

$$
\begin{array}{r}
\Lambda=\Lambda_{0}+\sum_{k_{s}=1}^{\infty} \Lambda_{k_{s}} \cos \left(k_{s} Z_{s} \alpha_{s}\right)+\sum_{k_{r}=1}^{\infty} \Lambda_{k_{r}} \cos \left(k_{r} Z_{r}\left(\alpha_{s}-\alpha_{r}\right)\right)+\frac{1}{2} \sum_{k_{s}=1}^{\infty} \sum_{k_{r}=1}^{\infty} \Lambda_{k_{s} k_{r}}\left\{\cos \left(\left(k_{s} Z_{s}-k_{r} Z_{r}\right) \alpha_{s}+k_{r} Z_{r} \alpha_{r}\right)\right. \\
\left.+\cos \left(\left(k_{s} Z_{s}+k_{r} Z_{r}\right) \alpha_{s}-k_{r} Z_{r} \alpha_{r}\right)\right\}
\end{array}
$$

where

$$
\Lambda_{0}=\mu_{0} A^{0} \quad \Lambda_{k_{s}}=2 \mu_{0} A^{s} f\left(k_{s}\right) \quad \Lambda_{k_{r}}=2 \mu_{0} A^{r} f\left(k_{r}\right) \quad \Lambda_{k_{s} k_{r}}=4 \mu_{0} A^{s r} f\left(k_{s}\right) f\left(k_{r}\right)
$$

and

$$
\begin{gathered}
f\left(k_{s}\right)=\frac{\sin \left(\pi k_{s} r_{d}^{s}\right)}{2 k_{s}} \quad f\left(k_{r}\right)=\frac{\sin \left(\pi k_{r} r_{d}^{r}\right)}{2 k_{r}} \\
A^{0}=\frac{1}{g_{M}}\left(1+\frac{p_{s}^{f} r_{d}^{s}}{g^{r}}+\frac{p_{r}^{f} r_{d}^{r}}{g^{s}}+\left(1+\frac{g_{M}}{g}\right) \frac{p_{s}^{f} r_{d}^{s}}{g^{r}} \frac{p_{r}^{f} r_{d}^{r}}{g^{s}}\right) \\
A^{s}=\frac{2 p_{s}^{f}}{\pi g_{M} g^{r}}\left(1+\left(1+\frac{g_{M}}{g}\right) \frac{p_{r}^{f} r_{d}^{r}}{g^{s}}\right) \quad A^{r}=\frac{2 p_{r}^{f}}{\pi g_{M} g^{s}}\left(1+\left(1+\frac{g_{M}}{g}\right) \frac{p_{s}^{f} r_{d}^{s}}{g^{r}}\right) \quad A^{s r}=\frac{4 p_{r}^{f} p_{s}^{f}}{\pi^{2} g_{M} g^{s} g^{r}}\left(1+\frac{g_{M}}{g}\right) \\
g_{M}=g+p_{s}^{f}+p_{r}^{f} g^{s}=g+p_{s}^{f} \quad g^{r}=g+p_{r}^{f}
\end{gathered}
$$

$g$ is the minimal effective air-gap width whereas $g_{M}$ is the maximal effective air-gap width. $\alpha_{r}$ is the angular position of rotor bar number 1 in stator steady frame:

$$
\alpha_{r}(t)=\frac{\omega_{1}}{p}(1-s) t+\alpha_{r}^{0}
$$

$p_{s}^{f}$ and $p_{r}^{f}$ are the stator and rotor fictitious slot depths, their values are fixed as suggested by (Brudny 1997) proportionally to rotor and stator slot openings $\left(l_{r e} / 5\right.$ and $\left.l_{s e} / 5\right) . r_{d}^{s}$ and $r_{d}^{r}$ are stator and rotor slotting ratios

$$
r_{d}^{s}=l_{s d} /\left(l_{s d}+l_{s e}\right) \quad r_{d}^{r}=l_{r d} /\left(l_{r d}+l_{r e}\right)
$$

Expression (26) allows to easily identify permeance waves orders and frequencies. They are reported in Table II where notation $f_{R}=f_{1}(1-s) / p$ is used. When two waves of frequencies and orders $\left(f_{1}, m_{1}\right)$ and $\left(f_{2}, m_{2}\right)$ are multiplied, they generate two aditionnal waves $\left(f_{1}+f_{2}, m_{1}+m_{2}\right)$ and $\left(f_{1}-f_{2}, m_{1}-m_{2}\right)$. These new waves can be represented by $\left(f_{1}+\eta_{12} f_{2}, m_{1}+\eta_{12} m_{2}\right)^{2}$, where the symbol $\eta_{12}$ can either take the value 1 or -1 . Using that symbol instead of \pm 1 makes it easier to associate a given frequency to its spatial order.

In Table II, $P_{0}$ stands for the mean permeance $\Lambda_{0}$, whereas $P_{s}$ and $P_{r}$ represent the stator and rotor slotting contributions, and $P_{s r}$ their interaction. In this work, $P_{s r}$ waves will not be considered for readability purpose. Saturation and eccentricity harmonics could be added in this table.

${ }^{2}$ Using that notation, the waves $(m, f)$ and $(-m,-f)$ are the same. 
TABLE II

PERMEANCE WAVES FREQUENCIES AND SPATIAL ORDERS

\begin{tabular}{|c|c|c|c|}
\hline Name/Amplitude & Spatial orders & Frequencies & Comments \\
\hline \hline$P_{0}$ & 0 & 0 & \\
$P_{s}$ & $k_{s} Z_{s}$ & 0 & $k_{r}, k_{s} \geq 1$ \\
$P_{r}$ & $k_{r} Z_{r}$ & $-k_{r} Z_{r} f_{R}$ & \\
$P_{s r}$ & $k_{s} Z_{s}+\eta k_{r} Z_{r}$ & $-\eta k_{r} Z_{r} f_{R}$ & \\
\hline
\end{tabular}

\section{B. Stator mmf orders and frequencies}

The same analysis can be carried with mmfs. Stator mmf is the product of stator currents of frequencies $f_{n}^{s}$ with stator TFs. Stator TFs do not depend on time, and therefore only bring space harmonics $\nu_{s}$. Their expression could be obtained from the Fourier expansion of the stator turn function $N_{0}^{s}$ illustrated in FIG. 2, as it is going to be done for rotor in section V-C:

$$
N_{k}^{s}\left(\alpha_{s}\right)=\sum_{n_{s}=1}^{\infty} \frac{2}{n_{s}^{2} \beta_{s}\left(\pi-\beta_{s}\right)} \sin \left(n_{r}\left(\alpha_{s}-\alpha_{s}^{k}\right)\right)
$$

where $\alpha_{s}^{k}$ is the $k$-th slot angular position:

$$
\alpha_{s}^{k}=\alpha_{s}^{0}+(k-1) \frac{2 \pi}{Z_{s}}
$$

However, we are here going to use the work of WACH (Wach 1998) who detailed the space harmonic content of any fractionalslot winding. As the studied machine is double-layer wound, the number of coils per pole and phase $m_{c}$ equals the number of slots per pole and phase $m_{s}$

$$
m_{s}=m_{c}=\frac{Z_{s}}{2 p q_{s}}=\frac{27}{12}=\frac{9}{4}=2+\frac{1}{4} \doteq I_{c}+\frac{i}{h}
$$

where $I_{c}$ is the integer part of $m_{s}$ reduced improper fraction. As $h=4$ is even, space harmonics $\nu_{s}$ generated by stator TF are given by (Wach 1998)

$$
\nu_{s}=k \frac{2 p}{h}=k \quad k \in \mathbb{N}^{*}
$$

Finally, as the number of phases $q_{s}=3$ is prime and odd, the total sum of phase TFs does not contain space harmonics multiple of $q_{s}$. Space harmonics brought by the stator mmf in the air-gap are then

$$
\nu_{s}=1,2,4,5,7, \ldots=\left|p+\epsilon_{s} q_{s} h_{s}\right| \quad \epsilon_{s}= \pm 1, h_{s} \in \mathbb{N}
$$

These theoretical results agree with the simulation presented in Fig. 4. In that figure, the real Fourier transform is used which does not allow to distinguish the propagation direction of space harmonic fields. For instance, the space harmonic $\nu_{s}=\left|p-q_{s}\right|=1$ for $h_{s}=1$ rotates backward whereas the stator $\mathrm{mmf}$ fundamental space harmonic given by $\nu_{s}=p=2$ for $h_{s}=0$ rotates forward. Integral windings only bring space harmonics of the form $\left|\epsilon_{s} p+2 p q_{q} h_{q}\right|=p, 5 p, 7 p, 11 p \ldots$.

\section{Rotor mmf orders and frequencies}

Rotor mmf $f_{m m}^{r}$ is the product of the rotor currents with rotor turns functions $N_{k}^{r}$ :

$$
f_{m m}^{r}\left(t, \alpha_{s}\right)=\sum_{k=1}^{Z_{r}} \sum_{m, n} I_{m n}^{r} \sin \left(s_{m n} \omega_{n} t-m k \frac{2 \pi}{Z_{r}}+\phi_{m n}^{r}\right) N_{k}^{r}\left(t, \alpha_{s}\right)
$$

where $m=\nu_{s}$ stands for the stator winding space harmonics induced in rotor bars (Henao et al. 2005), $I_{m n}^{r}$ is the maximum rotor bars current computed by the aid of the equivalent circuit described in section II-A and $\phi_{m n}^{r}$ its phase angle. Rotor TF spectrum is more complex than stator one because it brings both time and space harmonics. TF associated to a single bar is plotted in Fig. 7 in stator steady frame.

In a rotor tooth centered frame, this function is even and its Fourier series is

$$
N_{0}^{r}\left(\alpha_{s}\right)=\frac{\beta_{r}}{2 \pi}\left(\frac{1}{Z_{r}}-\frac{1}{2}\right)+\sum_{n_{r}=1}^{\infty} A_{n_{r}} \cos \left(n_{r} \alpha_{s}\right)
$$

where

$$
A_{n_{r}}=\frac{2}{n_{r} \pi}\left(\frac{2}{\beta_{r} n_{r}} \sin \left(\frac{n_{r} \pi}{Z_{r}}\right) \sin \left(n_{r} \beta_{r}\right)-\sin \left(n_{r}\left(\frac{\pi}{Z_{r}}+\beta_{r}\right)\right)\right)
$$




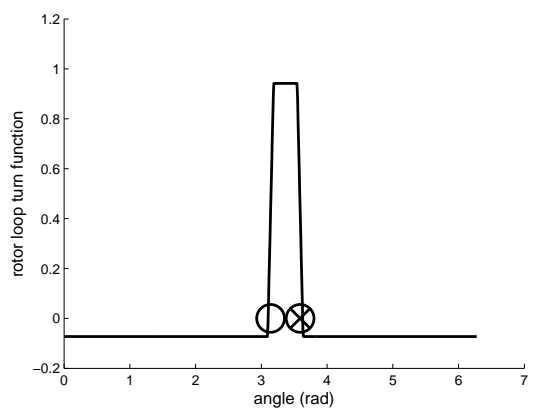

Fig. 7. Single rotor bar turns function (TF) in function of $\alpha_{s}$ angle, including a linear rise in rotor slot of angular width $2 \beta_{r}$.

and $\beta_{r}$ is the half rotor slot angular opening. The term in $1 / n_{r}^{2}$ comes from the linear rise in slots, it vanishes when $\beta_{r} \rightarrow 0$. Note that the mean value $\frac{\beta_{r}}{2 \pi}\left(\frac{1}{Z_{r}}-\frac{1}{2}\right)$ will disappear when summing on rotor bars in rotor mmf expression (33). The angular position of the $k$-th bar $\alpha_{k}$ in the stator steady frame can be written under the form

$$
\alpha_{r}^{k}(t)=\alpha_{r}(t)+(k-1) \frac{2 \pi}{Z_{r}}
$$

$k$-th rotor tooth position $\theta_{r}^{k}$ is then given by

$$
\theta_{r}^{k}(t)=\frac{1}{2}\left(\alpha_{r}^{k+1}(t)+\alpha_{r}^{k}(t)\right)=\alpha_{r}(t)+(2 k-1) \frac{\pi}{Z_{r}}
$$

Finally, the $k$-th bar turns function is expressed as

$$
N_{k}^{r}\left(t, \alpha_{s}\right)=N_{0}^{r}\left(\alpha_{s}-\theta_{r}^{k}(t)\right)
$$

In (34), it seems that rotor TF brings all the space harmonics $n_{r}=1,2,3 \ldots$. However, when multiplying by rotor currents and summing on rotor bars mmfs, only the space harmonics $n_{r}=h_{r} Z_{r} \pm \nu_{s}=\nu_{r}$ have a non-zero contribution. In (33), one can see that the rotor currents of frequency $f_{\nu_{s} n}^{r}=s_{\nu_{s} n} \omega_{n}$ combine with the rotor TF frequencies $\nu_{r} f_{1}(1-s) / p$ and spatial orders $\nu_{r}$. The resulting spectrum, as well as stator's one, is summarized in Table III.

TABLE III

ROTOR AND STATOR MMF FREQUENCIES AND ASSOCIATED SPATIAL ORDERS

\begin{tabular}{|c|c|c|c|}
\hline Name/Amplitude & Spatial orders & Frequencies & Comments \\
\hline \hline$F_{s}$ & $\nu_{s}=p+\epsilon_{s} q_{s} h_{s}$ & $\epsilon_{s} f_{n}^{s}$ & $h_{s} \geq 0$ \\
$F_{r}$ & $\nu_{r}=h_{r} Z_{r}+\epsilon_{r} \nu_{s}$ & $\nu_{r} f_{R}+\epsilon_{r} f_{\nu_{s} n}^{r}$ & $h_{r} \geq 0$ \\
\hline
\end{tabular}

\section{Flux density orders and frequencies}

Air-gap flux density is the product of permeance waves of type $P_{0}, P_{s}$ and $P_{r}$ with mmf waves of type $F_{s}$ and $F_{r}$, its spectrum is given by their 6 possible combinations (Table IV).

TABLE IV

FLUX DENSITY FREQUENCIES AND ASSOCIATED SPATIAL ORDERS

\begin{tabular}{|c|c|c|}
\hline Name/Amplitude & Spatial orders & Frequencies \\
\hline \hline$P_{0} F_{s}$ & $\eta_{0 s} \nu_{s}$ & $\eta_{0 s} \epsilon_{s} f_{n}^{s}$ \\
$P_{0} F_{r}$ & $\eta_{0 r} \nu_{r}$ & $\eta_{0 r}\left(\nu_{r} f_{R}+\epsilon_{r} f_{\nu_{s} n}^{r}\right)$ \\
$P_{s} F_{s}$ & $k_{s} Z_{s}+\eta_{s s} \nu_{s}$ & $\eta_{s s} \epsilon_{s} f_{n}^{s}$ \\
$P_{s} F_{r}$ & $k_{s} Z_{s}+\eta_{s r} \nu_{r}$ & $\eta_{s r}\left(\nu_{r} f_{R}+\epsilon_{r} f_{\nu_{s} n}^{r}\right)$ \\
$P_{r} F_{s}$ & $k_{r} Z_{r}+\eta_{r s} \nu_{s}$ & $-k_{r} Z_{r} f_{R}+\eta_{r s} \epsilon_{s} f_{n}^{s}$ \\
$P_{r} F_{r}$ & $k_{r} Z_{r}+\eta_{r r} \nu_{r}$ & $-k_{r} Z_{r} f_{R}+\eta_{r r}\left(\nu_{r} f_{R}+\epsilon_{r} f_{\nu_{s} n}^{r}\right)$ \\
\hline
\end{tabular}




\section{E. Radial force orders and frequencies}

Finally, to obtain the electromagnetic force spectrum, one has to multiply all the flux density waves of Table IV one with another. Resulting lines are presented in Table $\mathrm{V}$ where high spatial order lines (e.g. the interaction of wave $P_{s} F_{s}$ with itself which leads to a $2 k_{s} Z_{s} \pm 2 \nu_{s}$ order ${ }^{3}$ ) and redundant lines like the interactions $P_{s} F_{s} P_{r} F_{r}$ and $P_{s} F_{r} P_{r} F_{s}$ have been removed. As these main lines are expressed in function of stator and rotor space harmonics $\nu_{s}$ and $\nu_{r}$, their expressions are general and can be applied to any stator and rotor winding.

\section{TABLE V}

MAIN RADIAL FORCE LINES FREQUENCIES AND SPATIAL ORDERS. FOR INSTANCE, $\epsilon_{r}=-\epsilon_{s}=1, \nu_{s}=\nu_{r}=p, k_{r}=k_{s}=1$ AND $\eta_{r}=-\eta_{s s}=1$ GIVE A FORCE LINE $L_{3}$ OF ORDER $Z_{s}-Z_{r}-2 p=2$ AND FREQUENCY $\left(Z_{r}-p\right) f_{R}+f_{1}-s f_{1}=Z_{r} f_{1}(1-s) / p$.

\begin{tabular}{|c|c|c|}
\hline Name/Amplitude & Spatial orders & Frequencies \\
\hline \hline$L_{1}=P_{s} F_{s} P_{s} F_{r}$ & $\eta_{s s} \nu_{s}-\eta_{s r} \nu_{r}$ & $\eta_{s s} \epsilon_{s} f_{n_{1}}^{s}-\eta_{s r}\left(\nu_{r} f_{R}+\epsilon_{r} f_{\nu_{s}}^{r} n_{2}\right)$ \\
$L_{2}=P_{s} F_{s} P_{r} F_{s}$ & $k_{s} Z_{s}-k_{r} Z_{r}+\nu_{s} \eta_{s s}-\nu_{s}^{\prime} \eta_{r s}$ & $k_{r} Z_{r} f_{R}+f_{n_{1}}^{s} \epsilon_{s} \eta_{s s}-f_{n_{2}}^{s} \epsilon_{s}^{\prime} \eta_{r s}$ \\
$L_{3}=P_{s} F_{s} P_{r} F_{r}$ & $k_{s} Z_{s}-k_{r} Z_{r}+\eta_{s s} \nu_{s}-\eta_{r r} \nu_{r}$ & $\left(k_{r} Z_{r}-\eta_{r r} \nu_{r}\right) f_{R}+\epsilon_{s} \eta_{s s} f_{n_{1}}^{s}-\epsilon_{r} \eta_{r r} f_{\nu_{s} n_{2}}^{r}$ \\
$L_{4}=P_{s} F_{r} P_{r} F_{r}$ & $k_{s} Z_{s}-k_{r} Z_{r}+\eta_{s r} \nu_{r}^{\prime}-\eta_{r r} \nu_{r}$ & $f_{R}\left(k_{r} Z_{r}+\eta_{s r} \nu_{r}^{\prime}-\eta_{r r} \nu_{r}\right)+\epsilon_{r} \eta_{s r} f_{\nu_{s} n_{1}}^{r}-\epsilon_{r}^{\prime} \eta_{r r} f_{\nu_{s} n_{2}}^{r}$ \\
$L_{5}=P_{r} F_{s} P_{r} F_{r}$ & $\eta_{r s} \nu_{s}-\eta_{r r} \nu_{r}$ & $\eta_{r s} \epsilon_{s} f_{n_{1}}^{s}-\eta_{r r}\left(\nu_{r} f_{R}+\epsilon_{r} f_{\nu_{s}}^{r} n_{2}\right)$ \\
$L_{6}=P_{0} F_{s} P_{0} F_{s}$ & $\eta_{0 s} \nu_{s}-\eta_{0 s}^{\prime} \nu_{s}^{\prime}$ & $\epsilon_{s} \eta_{0 s} f_{n_{1}}^{s}-\epsilon_{s}^{\prime} \eta_{0 s}^{\prime} f_{n_{2}}^{s}$ \\
$L_{7}=P_{0} F_{r} P_{0} F_{r}$ & $\eta_{0 r} \nu_{r}-\eta_{0 r}^{\prime} \nu_{r}^{\prime}$ & $f_{R}\left(\eta_{0 r} \nu_{r}-\eta_{0 r}^{\prime} \nu_{r}^{\prime}\right)+\epsilon_{r} \eta_{0 r} f_{\nu_{s} n_{1}}^{r}-\epsilon_{r}^{\prime} \eta_{0 r}^{\prime} f_{\nu_{s} n_{2}}^{r}$ \\
$L_{8}=P_{0} F_{r} P_{0} F_{s}$ & $\eta_{0 r} \nu_{r}-\eta_{0 s} \nu_{s}$ & $\eta_{0 r}\left(\nu_{r} f_{R}+\epsilon_{r} f_{\nu_{s} n_{1}}^{r}-\epsilon_{s} \eta_{0 s} f_{n_{2}}^{s}\right.$ \\
\hline
\end{tabular}

In sinusoidal case, $f_{n}^{s}=f_{1}$, and as a consequence lines $L_{1}, L_{5}, L_{6}, L_{7}$ and $L_{8}$ have low frequencies (typically $2 f_{1} \leq 200$ $\mathrm{Hz})$ and might be covered by mechanical noise. Other lines associated to the fundamental $\mathrm{mmfs}\left(\nu_{s}=\nu_{r}=p, \epsilon_{s}=-1\right.$, $\epsilon_{r}=1$ and $f_{\nu_{s} 1}^{r}=s f_{1}$ ) have all the following form:

$$
F_{2,3,4}=f_{1}\left((1-s) \frac{k_{r} Z_{r}}{p} \pm\left.\right|_{0} ^{2}\right)
$$

They are associated to spatial orders of the form

$$
M_{2,3,4}= \pm k_{s} Z_{s} \mp k_{r} Z_{r} \pm\left.\right|_{0} ^{2 p}
$$

Among these lines, the most important ones are $L_{2}$ because they do not involve rotor mmf waves of amplitude $F_{r} \ll F_{s}$. These lines are mostly responsible for electromagnetic noise in sinusoidal case, because they are associated to low spatial orders (Jordan 1950). Notice that such lines, as they are caused by fundamental current, generally remain in case of a non-sinusoidal supply.

In non-sinusoidal case, the force lines $L_{1}, L_{5}, L_{6}, L_{7}$ and $L_{8}$ can be located at high frequencies and therefore significantly contribute to acoustic noise. We can see that they are necessarily associated to orders

$$
M_{1,5,6,7,8}=0 \quad \text { or } 2 p
$$

when considering the mmf space fundamentals. Among them, the lines of highest amplitude are of the form $L_{6}$ and occur at frequencies $F_{6}=\left| \pm f_{1} \pm f_{n}^{s}\right|$. Lines $L_{7}$ may be neglictible as they involve the square amplitude of rotor mmf waves and low frequencies. Other important lines in PWM case are therefore $L_{1}$ and $L_{5}$, which have exactly the same form of orders and frequencies, and $L_{8}$. Among them, the main lines are obtained taking the fundamental time harmonic of rotor current, and the corresponding frequencies are therefore also of the form (Lo, Chan, $\mathrm{Zhu}, \mathrm{Xu}, \mathrm{Howe} \&$ Chau 2000)

$$
F_{1,5,6,8}=\left| \pm f_{1} \pm f_{n}^{s}\right|
$$

\section{Vi. Simulation Results}

\section{A. Simulated spectra}

A first simulation was run at nominal frequency $f_{1}=50 \mathrm{~Hz}$, without considering the stator space harmonics induced in rotor currents. The corresponding A-weighted noise spectrum on the whole audible range [0 Hz, $20 \mathrm{kHz}]$ is displayed in Fig. 8. Note that such a precise and wide spectrum could not be obtained with finite element (for vibrations) and boundary element methods (for sound pressure). These numerical methods can hardly compute a noise spectrum up to $3200 \mathrm{~Hz}$, and with a prohibitive computational time of several hours, whereas the analytical model of DivA runs in a few seconds on a $2 \mathrm{GHz}$ laptop.

Simulated sound power level reaches $58 \mathrm{dBA}$ : as it was observed during tests, the machine is not very noisy because no magnetic line really emerges from the spectrum at this particular speed and in sinusoidal case.

\footnotetext{
${ }^{3} \mathrm{~A}$ high $\nu_{s}$ and a low $k_{s}$, e.g. $k_{s}=1$, could result in a low spatial order. However, the higher $\nu_{s}$ is, the lower the amplitude of stator mmf wave $F_{s}$ is.
} 


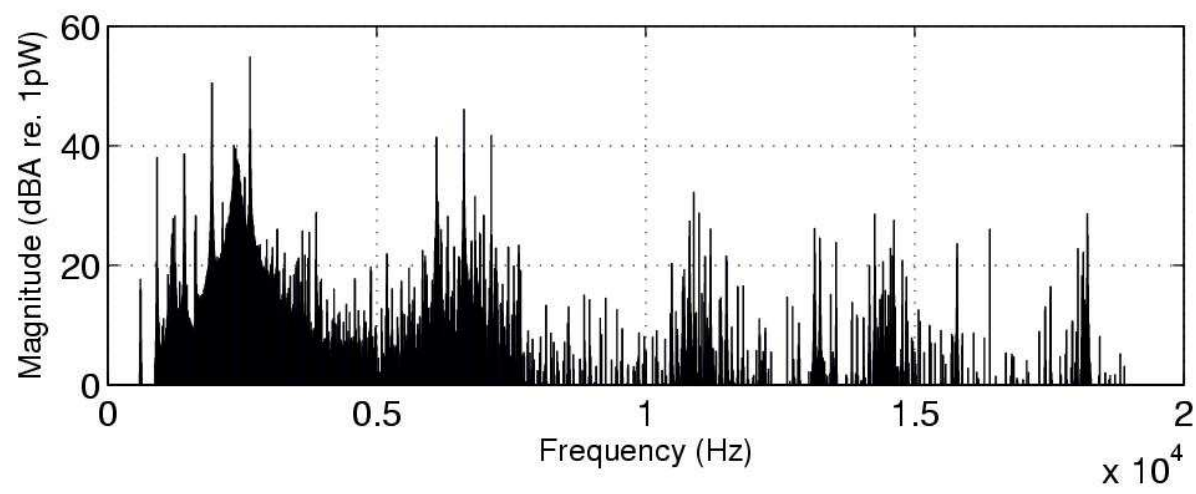

Fig. 8. Simulated A-weighted audible electromagnetic noise $L_{w A}(\mathrm{dBA})$ in on-load sinusoidal case $\left(f_{1}=50 \mathrm{~Hz}, s=3.05 \%\right)$

In order to properly interpretate these lines, it can be useful to find their associated spatial order. It can be done using the analytical results of previous section, but numerically it is also possible to compute each spectrum line without taking into account the acoustic power radiated by a particular spatial mode, and then quantify the contribution of each spatial mode to each spectrum line. This contribution is plotted in Fig. 9 in the range [500 Hz, $3000 \mathrm{~Hz}]$ where the highest vibration lines appear.

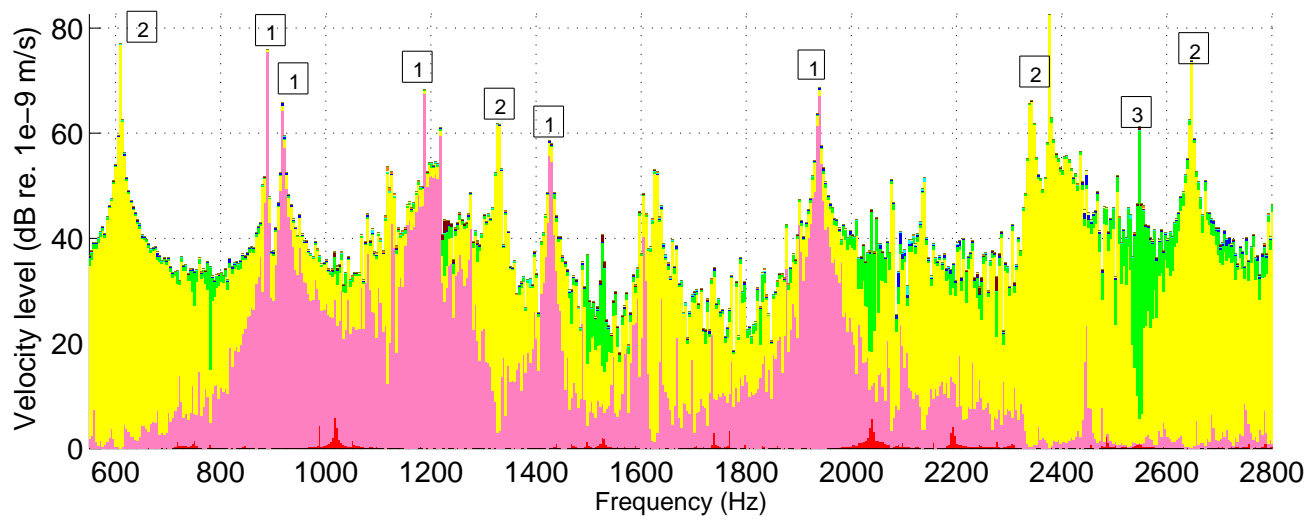

Fig. 9. Simulated vibration velocity spectrum with spatial mode contribution of each line $\left(f_{1}=50 \mathrm{~Hz}, s=3.05 \%\right)$. The numbers indicate the spatial orders of vibration lines.

We can see that the main lines have mode numbers $m=1,2$ and 3. For instance, a line of order 1 occurs at $1935 \mathrm{~Hz}$. Order number 1 can be obtained with $1=3 Z_{s}-4 Z_{r}+2 p$ i.e. $k_{r}=4$. We can see that the $1935 \mathrm{~Hz}$ frequency precisely corresponds to the form $F_{2}=f_{1}\left(4(1-s) \frac{Z_{r}}{p}-2\right)$ for $\eta_{s s}=1$ and $\eta_{r s}=-1$. In the same way, a line of order $2=Z_{s}-Z_{r}-2 p$ occurs at $610 \mathrm{~Hz}$ which corresponds to $F_{2}=f_{1}\left((1-s) \frac{Z_{r}}{p}+2\right) \mathrm{Hz}\left(k_{r}=4, \eta_{s s}=-1\right.$ and $\left.\eta_{r s}=1\right)$. Finally, a line of order $3=4 Z_{s}-5 Z_{r}$ occurs at $2550 \mathrm{~Hz}$ of the form $F_{2}=f_{1}\left(5(1-s) \frac{Z_{r}}{p}\right)\left(k_{r}=5, \eta_{s s}=\eta_{r s}=1\right)$. These few examples show how the simulations results correctly fit to previous analytical results.

Modal contribution analysis can be also carried with sound power level $L_{w}$ at variable speed (Fig. 10), that is to say during a motor speed ramp at constant flux. Sound power level is computed by simulating nearly one rotor turn, so if the speed do not significantly vary during this turn (which is a reasonable assumption in our case), the variable-speed level can be obtained by computing the sound power level at each speed step.

Such a graph allows for instance to see that around supply frequency $f_{1}=30 \mathrm{~Hz}$ it is mode $m=1$ which radiate the most, whereas at $f_{1}=45 \mathrm{~Hz}$ it is $m=2$. The $30 \mathrm{~Hz}$ resonance comes from the match between the magnetic line $f_{1}\left(4(1-s) Z_{r} / p-2\right)$ which has been previously pointed out and the $1200 \mathrm{~Hz}$ mode number 1 natural frequency.

Previous simulations show that some odd spatial modes $(m=1,3)$ play an important role in noise radiation. Usually, induction machines have even slot numbers: consequently, $\overline{Z_{r}}=\overline{Z_{s}}=\overline{0}(\bar{n}$ stands for the congruence modulo 2 operator, i.e. $\bar{n}=\overline{0}$ if $n$ is even and $\bar{n}=\overline{1}$ otherwise). In that case, winding is integral and $\nu_{s}=p\left(2 q_{s} h_{s}+\epsilon_{s}\right)$ so that $\bar{\nu}_{s}= \pm \bar{p}$. Therefore, all the deflection modes parity are given by

$$
\bar{M}= \pm \bar{k}_{s} \bar{Z}_{s} \mp \bar{k}_{r} \bar{Z}_{r} \pm \mid \bar{\nu}_{s} \pm \bar{\nu}_{r}=\overline{0}
$$




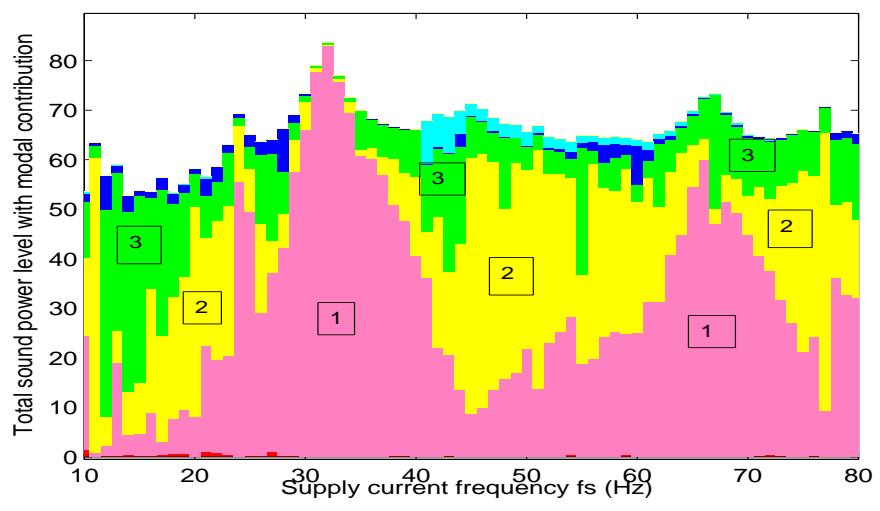

Fig. 10. Variable-speed simulation with spatial orders contribution to total sound power level $L_{w}(\mathrm{~dB})$. The numbers indicate the spatial orders.

which means that the only spatial modes that are excited by electromagnetic vibration are even. When using odd numbers of rotor and stator slots, which necessarily impose a fractional-slot winding as $Z_{s} /\left(2 p q_{s}\right)$ cannot be integral, we do not necessarily have $\bar{M}=\overline{0}$ : the electromagnetic power brought by Maxwell air-gap pressure can dissipate as vibrations through all the stator deflection modes. Therefore, acoustic power is more uniformly distributed in spatial modes and if a resonance occur, it will be smaller than in traditonal even slot number combination machines. However, it also means that electromagnetic pressure can excite some odd modes natural frequency. This it is not a matter of windings but of slot number parity.

\section{CONCLUSION}

An analytical noise-predictive model of the induction machine and its converter was presented. The expression of main noise spectrum lines frequencies and spatial orders, including stator winding and rotor bars space harmonics, as well as PWM time harmonics, was derived analytically. These theoretical results were favorably compared to simulations and expriments in on-load sinusoidal case.

Saturation and eccentricity effects have not been discussed in that article, although they are taken into account in DIVA. Saturation modify the air-gap flux density shape, which adds new harmonics in Maxwell forces spectrum and modify noise spectrum. This new harmonic content can be modelled by adding saturation permeance waves (Maliti 2000). Dynamic and static eccentricities also change permeance waves, and can be easily taken into account (Toliyat \& Arefeen 1996) without increasing computational cost.

Future work will address the effect of PWM on magnetic noise generation, and detail other experimental validations of the developed simulation tool.

\section{REFERENCES}

Ait-Hammouda, A. (2005), Prédimensionnement et étude de sensibilité vibro-acoustique de machines courant alternatif et vitesse variable, PhD thesis, Université des Sciences et des Technologies de Lille, France.

Besnerais, J. L., Fasquelle, A., Hecquet, M., Lanfranchi, V. \& Brochet, P. (2006), A fast noise-predictive multiphysical model of the PWM-controlled induction machine, in 'Proc. of the International Conference on Electrical Machines (ICEM'06)', Chania, Greece.

Bossio, G., Angelo, C. D., Solsona, J., Garcia, G. \& Valla, M. (2004), 'A 2-D model of the induction machine: an extension of the modified winding function approach', IEEE Trans. on Energy Conversion 19(1).

Brudny, J. (1997), 'Modélisation de la denture des machines asynchrones : phénomènes de résonances', Journal of Physics III 37(7).

Cremer, L., Heckl, M. \& Ungar, E. (1988), Structure-Borne Sound, Springer-Verlag.

Henao, H., Razik, H. \& Capolino, G. (2005), 'Analytical approach of the stator current frequency harmonics computation for detection of induction machine rotor faults', IEEE Trans. on Ind. App. 41(3).

Hesse, H. (1992), 'Air gap permeance in doubly-slotted asynchronous machines', IEEE Trans. on Energy Conversion 7(3).

Hubert, A. (2000), Contribution l'étude des bruits acoustiques générés lors de l'association machines électriques - convertisseurs statiques de puissances application la machine asynchrone, $\mathrm{PhD}$ thesis, Université des Technologies de Compiègne, France.

Hubert, A. \& Friedrich, G. (2002), 'Influence of power converter on induction motor acoustic noise: interaction between control strategy and mechanical structure', Electric Power Applications, IEE Proceedings 149.

Joksimovic, G., Djurovic, M. \& Penman, J. (2001), 'Cage rotor MMF : winding function approach', IEEE Power Engineering Review 21(4).

Jordan, H. (1950), Electric motor silencer - formation and elimination of the noises in the electric motors, W. Giradet-Essen editor.

Lo, W., Chan, C., Zhu, Z., Xu, L., Howe, D. \& Chau, K. (2000), 'Acoustic noise radiated by PWM-controlled induction machine drives', IEEE Trans. on Industrial Electronics 47(4).

Maliti, K. (2000), Modelling and analysis of magnetic noise in squirrel-cage induction motors, PhD thesis, Stockholm.

Matsuse, K., Hayashida, T., Kubota, H. \& Yoshida, T. (1994), 'Analysis of inverter-fed high speed induction motor considering crosspath resistance between adjacent rotor bars', IEEE Trans. Ind. Appl. 30(3). 
Salminen, P. (2004), Fractional slot permanent magnet synchronous motors for low speed application, PhD thesis, Lappeenranta University of Technology, Finland.

Timar, P. \& Lai, J. (1994), 'Acoustic noise of electromagnetic origin in an ideal frequency-converter-driven induction motor', IEE Proceedings on Electrical Power Applications 141(6).

Toliyat, A. \& Arefeen, M. (1996), 'A method for dynamic simulation of air-gap eccentricity in induction machines', IEEE Trans. on Industry Applications 32(4).

Verma, S. \& Balan, A. (1998), 'Experimental investigations on the stators of electrical machines in relation to vibration and noise problems', IEE Proceedings on Electrical Power Applications 145(5).

Wach, P. (1998), 'Algorithmic method of design and analysis of fractional-slot windings of AC windings', Electrical Engineering 81(3).

Yang, S. J. (1981), Low noise electrical motors, Clarendon Press, Oxford. 